\title{
Evaluation of a Public Transport Priority Methodology
}

\author{
$\mathrm{Li} \mathrm{Lin}^{1, \text { a }}$, YongKang Zhang ${ }^{2, \mathrm{~b}}$ and Zhuang $\mathrm{Hu}^{3, \mathrm{c}}$ \\ ${ }^{1}$ Automobile and Traffic Engineering College, Nanjing Forestry University, Nanjing, China. \\ Corresponding author. Tel.: +86 13705180025 \\ ${ }^{2}$ Automobile and Traffic Engineering College, Nanjing Forestry University, Nanjing, China. \\ Corresponding author. Tel.: +86 18013970421 \\ ${ }^{3}$ Automobile and Traffic Engineering College, Nanjing Forestry University, Nanjing, China. \\ Corresponding author. Tel.: +86 18020105226

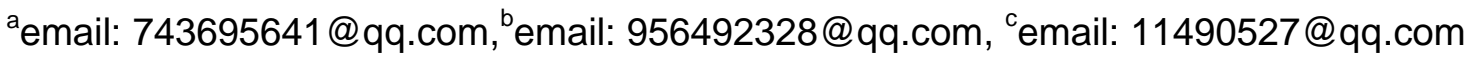

\begin{abstract}
Keywords: signal control; public transport priority; traffic-responsive urban control.
Abstract. Compared with the past decades, the methodologies of bus priority at traffic signals is being researched more and more extensively because of the strong development of public transport. Several methods have been used to attract people to use public transport. These methods may be divided into two categories: facility-design-based measures and signal-control-based measures. In this paper, a signal-control-based methodology is presented and implemented in a microscopic simulation environment emulating the network of Nanjing, China, with realistic traffic conditions. The objective of the control is to reduce the average delay of buses without creating major disturbances to the rest of the network. In addition to a fixed-time strategy combined with public transport priority (PTP), the traffic-responsive urban control (TUC) strategy with PTP is implemented in order to reduce the buses delays.
\end{abstract}

\section{Introduction}

Most of urban road networks are facing serious traffic problems, which are the result of the growth of population in the cities and the old road networks. Many cities did not expect to the development during the construction of the network, many factors have not been considered, such as adequate parking space or space for exclusive bus lanes. As a consequence, the most serious problems that are noticed are traffic congestion, increased travel time of private and public vehicles and air pollution. The way that could be used to mitigate some of the above problems is the frequent use of public transport(PT) means. However, this needs to improve the public transport services, because the travel time of PT vehicles, especially of buses, is much longer than the one of private vehicles. The main reason for the long travel times of buses is that they face significant delays at each bus stop, for boarding and alighting. In addition, the bus must travel along the tortuous routes. Because of these factors cannot be controlled, the purpose of the researchers is to improve the movement of PT vehicles at signalized junctions.

This paper mainly introduces and evaluates a local real-time reactive rule-based PTP methodology. Specifically, PTP is used in real time to provide priority at signalized junctions so that the PT vehicle delays due to the red light are reduced. The priority is achieved by changing the signal settings without affecting the rules of road safety. In the case of multiple priority requests at the same junction and within the same period, a first-come-first-served policy is applied, unless a subsequent request is served by the same stage, in which case a priority is provided only by green extension. It is also important to verify that the strategy will not interfere with the rest of the network. For this reason, two signal control scenarios are considered and compared: first, the traffic signals are controlled with a fixed-time plan; second, the traffic-responsive urban control (TUC)strategy with PTP is also implemented.

The methodology is implemented in a microscopic simulation environment emulating the urban network of Nanjing, China, using realistic traffic conditions; only consider one bus lane, which 
excludes priority conflicts for buses being served at different stages. The results are evaluated based on two criteria: the average delay and the total travel time (TTT).

\section{Public transport priority methodology}

\section{PTP methodology}

The PTP methodology is a real-time, reactive, rule-based priority strategy. The cycle time of each junction is fixed, and the priority is achieved by green extension or red interruption. Instead of changing the order of the phase, the PT vehicle's green time is extended, if it is necessary, only by the other phases be compressed to the permitted minimum times.

Considering that a public transport vehicle has been detected to approach a signalized junction, the following steps are performed:

Step 1: the time of the bus from the detection point to the stop-line is calculated using an estimation of the average speed of the vehicle and the distance from the stop-line. The detection of the bus is performed either at the beginning of the link or at the moment that the bus is departing from a bus stop.

Step 2: If the travel time from step 1is enough for the bus to travel through the intersection, then there is no need to provide priority. Otherwise, according to the phase that will be executed at the junction, priority is given by choosing the most appropriate option: green extension or red interruption. If no priority is needed, the strategy considers the next request, but only for potential green extension.

\section{A traffic-responsive urban control strategy}

PTP control improves the travel time of the buses by changing the signal plan at each junction. However, it should be noticed that the effect of these control actions to the rest of the network. In congested networks, the travel time of private cars is already high, these changes on the signal plan in favour of the public transport vehicles may cause extra interference to the rest of the network and bigger queues and delays. For this reason, the traffic-responsive urban control (TUC) strategy is implemented to optimize the flow and the travel time of all the vehicles in the network.

The TUC strategy consists of three parts:

(1) Split control: Minimizes the risk of queue spillback and oversaturation.

In order to achieve the objective, split control approaches the urban traffic control problem as an LQ optimal control problem and varies suitably the green-phase duration of each stage without changing the cycle times or the offsets.

(2) Cycle control: adjust the cycle time of the network according to the observed maximum saturation level.

For each intersection, the cycle time affects the whole traffic network, because a long cycle time can improve the capacity of the intersection, but also increase the waiting time during the red phase. Therefore, the cycle time should be adjusted depending on the traffic conditions and this is effectuated in TUC by a feedback algorithm. the feedback algorithm increases or decreases the cycle time using as a criterion the current maximum saturation level.

(3) Offset control: Creates green waves, taking into account the possibility of existing vehicle queues.

This part of the TUC strategy is specifying the offset between successive junctions so that green waves are created in an arterial. To achieve this, offset control is performed in a decentralized way. For each couple of successive junctions, TUC changes the starting time of a specific stage of the upstream junction. TUC and the PTP methodology can be combined to optimize the travel times of buses and cars.

\section{Simulation and results}

\section{Network description}

For the implementation of the PTP methodology, a part of the network of Nanjing, China, has been modeled in the microscopic simulator AIMSUN 7.0. The network contains 15 signalized junctions, 
from which 6 can provide priority to the buses. The selected intersection is located at the center of the network, and the traffic volume of private and PT vehicles is very high.

The buses are traveling on the mixed lanes, Bus detectors have been installed at the upstream end of the signalized approaches. These detectors are used to detect buses and request priority from the PTP system installed locally for each junction. Bus passage measurements from these detectors are collected every second. The bus stop stations are arranged on upstream of these detectors to avoid any impact on the estimation of the arrival time at the junction's stop-line. The network is simulated using realistic dynamically varying demand for a period of four hours, from 14:00 to 18:00.

The simulation replications have been produced for each scenario. The results will be evaluated based on two criteria: the average delay and the average total travel time (TTT).

\section{Fixed-Time plan and PTP}

The first scenario is the one with a fixed-time plan and the PTP methodology. The case with PTP is compared with the case without priority control. and Tables 1-2 present the average results for this first scenario.

Table 1 shows that the average delay time of the buses is reduced by $9.64 \%$ with the PTP and the average delay time of all the vehicles in the network is reduced by $1.38 \%$ with the PTP, compared to the case with no priority control (NO-PTP).As a result, the conclusion of the first scenario is that the methodology serves effectively the priority requests without affecting significantly the overall traffic conditions in the network.

Table 1: Average delay time with a fixed-time plan and high-frequency priority requests

\begin{tabular}{|c|c|c|c|}
\hline $\begin{array}{c}\text { Delay } \\
(\mathrm{sec} / \mathrm{km})\end{array}$ & NO-PTP & PTP & $\begin{array}{c}\text { Change of PTP } \\
\text { compared to } \\
\text { NO-PTP }(\%)\end{array}$ \\
\hline Bus & 70.51 & 63.71 & -9.64 \\
\hline Car & 82.32 & 82.11 & -0.25 \\
\hline All & 81.56 & 80.43 & -1.38 \\
\hline
\end{tabular}

Table 2 shows that the average TTT of the buses is reduced by $11.84 \%$ with PTP,while the methodology manages to maintain the TTT of the cars and all the vehicles in the network, at almost the same level.

Table 2: Average TTT with a fixed-time plan

\begin{tabular}{|c|c|c|c|}
\hline $\begin{array}{c}\text { TTT } \\
\text { (hours) }\end{array}$ & NO-PTP & PTP & $\begin{array}{c}\text { Change of PTP } \\
\text { compared to } \\
\text { NO-PTP }(\%)\end{array}$ \\
\hline Bus & 1.52 & 1.34 & -11.84 \\
\hline Car & 492.1 & 492.8 & 0.14 \\
\hline All & 497.5 & 498.2 & 0.12 \\
\hline
\end{tabular}

\section{TUC and PTP}

The second scenario is used to study the effect of the PTP methodology when TUC is used for all the junctions of the network. The demands are the same with scenario 1.

Table 3 shows that the delay time of the buses is reduced by $6.06 \%$ with PTP compared to the TUC operation without PTP. The average delay time of the cars is increased by $0.65 \%$ with PTP. However, the delay time of the cars is reduced compared with Table 1. As a result, priority control continues reducing the delay time of the buses while TUC reduces the delay time of all the vehicles in the network. 
Table 3: Average delay time with TUC

\begin{tabular}{|c|c|c|c|}
\hline $\begin{array}{c}\text { Delay } \\
(\mathrm{sec} / \mathrm{km})\end{array}$ & NO-PTP & PTP & $\begin{array}{c}\text { Change of PTP } \\
\text { compared to } \\
\text { NO-PTP }(\%)\end{array}$ \\
\hline Bus & 65.33 & 61.37 & -6.06 \\
\hline Car & 73.53 & 74.01 & 0.65 \\
\hline All & 71.61 & 72.23 & 0.86 \\
\hline
\end{tabular}

Table 4 shows that the average TTT of the buses is reduced by $6 \%$ with PTP and the average TTT of the cars is increased by $0.37 \%$. However, comparing with the results of scenario 1 , we can conclude that the TTT of the cars is lower with TUC, even when applying priority control.

Table 4: Average TTT with TUC

\begin{tabular}{|c|c|c|c|}
\hline $\begin{array}{c}\text { TTT } \\
\text { (hours) }\end{array}$ & NO-PTP & PTP & $\begin{array}{c}\text { Change of PTP } \\
\text { compared to } \\
\text { NO-PTP (\%) }\end{array}$ \\
\hline Bus & 1.33 & 1.25 & -6 \\
\hline Car & 471.52 & 473.29 & 0.37 \\
\hline All & 469.32 & 470.11 & 0.16 \\
\hline
\end{tabular}

\section{Conclusions}

This section collects and compares the final results for scenarios 1 and 2. The aim is to conclude which is the most satisfactory priority option.

Table 5 shows the average delay of the fixed-time plan (FTP) and TUC. Comparing the TUC strategy with FTP, the average delay of the buses is reduced by $7.35 \%$,the average delay of the cars is reduced by $10.67 \%$ and the average delay overall is reduced by $12.19 \%$. This result shows that TUC is a real time strategy and is superior than fixed-time plans.

When applying PTP, comparing with no control, the average delay time of the buses is reduced by $9.64 \%$ with a fixed-time plan and by $6.06 \%$ with TUC. The delay time for the buses is $3.67 \%$ lower when using TUC instead of a fixed-time plan, while the delay time overall is $10.19 \%$ lower with TUC instead of a fixed-time plan. As a result, PTP is effective for each one of the cases considered while its combination with TUC is even more effective both for buses and cars.

Table 5: Comparison table for average delay time

\begin{tabular}{|c|c|c|c|}
\hline Delay (sec/km) & Vehicle Type & NO-PTP & PTP \\
\hline \multirow{3}{*}{ Fixed-Time Plan } & Bus & 70.51 & 63.71 \\
\hline & Car & 82.32 & 82.11 \\
\hline & All & 81.56 & 80.43 \\
\hline \multirow{3}{*}{ TUC } & Bus & 65.33 & 61.37 \\
\hline & Car & 73.53 & 74.01 \\
\hline & All & 71.61 & 72.23 \\
\hline \multirow{3}{*}{$\begin{array}{l}\text { Change for } \\
\text { TUC compared } \\
\text { to FTP }(\%)\end{array}$} & Bus & -7.35 & -3.67 \\
\hline & Car & -10.67 & -9.86 \\
\hline & All & -12.19 & -10.19 \\
\hline
\end{tabular}




\section{References}

[1] Awin, D.F., 2012. Integrating varieties of life course concepts. J. Gerontol. Ser. B Psychol. Sci. Soc. Sci. 67 (2), 206-220.

[2] Berg, J., Levin,L., Abramsson,M., Hagberg, J.-E., 2014. Mobility in the transition to retirement: the intertwining of transportation and everyday projects. J. Transp. Geogr. 38, 48-54.

[3] Boschmann, E.E., Brady, S.A., 2013. Travel behaviours, sustainable mobility, and transitoriented developments: a travel counts analysis of older adults in Denver, Colorado metropolitan area. J. Transp. Geogr. 33, 1-11.

[4] Cairns, S., Harmer, C., Hopkin, J., Skippon, S., 2014. Sociological perspectives on travel and mobilities: a review. Transp. Res. A 63, 107-117.

[5] Clark, B., Chatterjee, K., Melia, S., Knies, G., Laurie, H., 2014. Life events and travel behavior: exploring the interrelationship using UK household longitudinal study data. Transp. Res. Rec. 2413, 54-64.

[6] Frändberg, L., Vilhelmson, B., 2011. More or less travel: personal mobility trends in the Swedish population focusing gender and cohort. J. Transp. Geogr. 19, 1235-1244. 